\title{
Distribución gen fimA de Porphyromonas gingivalis en pacientes chilenos con periodontitis crónica
}

\section{Distribution of fimA genotypes of Porphyromonas gingivalis in Chilean patients with chronic periodontitis}

\author{
Miguel A. Rojas ${ }^{1 *}$, Jaime Jacques ${ }^{1}$, Sebastian Molinett ${ }^{2}$, Juliana N. Botelho ${ }^{1}$, Carlos Padilla ${ }^{3}$
}

1. Unidad de Periodoncia, Departamento de Estomatología, Universidad de Talca, Talca, Chile 2. Departamento de Biotecnología, Instituto de Ciencias Biológicas, Universidad de Talca, Talca, Chile 3. Departamento de Microbiología, Universidad de Talca, Talca, Chile

${ }^{*}$ Correspondencia a: Miguel Angel Rojas, DDS, MS, Unidad de Periodoncia, Departamento de Estomatología, Universidad de Talca 2 Norte 685 Talca, 3465548, Maule, Chile. | Tel: +56 071220452 | E-mail:mrojasc@utalca.cl

Trabajo recibido el 26/01/2017. Aprobado para su publicación el 05/09/2017

\section{RESUMEN}

Antecedentes: Gen fimA de Porphyromonas gingivalis es un importante factor de virulencia asociado al desarrollo y la progresión de periodontitis.

Objetivo: Cuantificar los niveles de $P$. gingivalis y la prevalencia de genotipos fimA en pacientes chilenos con diferentes grados de severidad de periodontitis crónica.

Metodología: Se analizaron 135 muestras subgingivales de 45 adultos (15 con leve, 15 con moderada y 15 con periodontitis severa) mediante qPCR para $P$. gingivalis y genotipos fimA (I-V and Ib).

Resultados: Se detectó $P$. gingivalis en el $73,3 \%$ de los pacientes con periodontitis crónica $(46,6 \%, 73,3 \%$ y $100 \%$ para las formas leve, moderada y severa, respectivamente). El gen fimA se detectó en el $66 \%$ de los sujetos positivos para $P$. gingivalis, siendo el fimA IV y I los genotipos más prevalentes. Además, se detectó fimA IV en el $75 \%$ y fimA I en el $62,5 \%$ de los casos severos y moderados de periodontitis, respectivamente. Los niveles aumentados de fimA IV se asociaron con periodontitis crónica severa.

Conclusiones: Los resultados sugieren una alta prevalencia de $P$. gingivalis y de sus genotipos fimA IV y I en pacientes con periodontitis crónica. Además fimA IV fue asociado con formas más severas de periodontitis crónica en esta población chilena.

PALABRAS CLAVE

Periodontitis crónica; Biofilm dental; Fimbrias bacterianas.

Rev. Clin. Periodoncia Implantol. Rehabil. Oral Vol. 10(3); 141-144, 2017.

\section{ABSTRACT}

Background: Porphyromonas gingivalis fimA gene is a key virulence factor and has been associated with development and progression of periodontal diseases.

Aim: To quantify the levels of $P$. gingivalis and the prevalence of fimA genotypes in Chilean patients with different severity of chronic periodontitis.

Methodology: One hundred and thirty five subgingival samples from 45 adults ( 15 with slight, 15 with moderate and 15 with severe chronic periodontitis, respectively) were analyzed by qPCR for $P$. gingivalis and fimA genotypes (I-V and Ib).

Results: P. gingivalis was detected in $73.3 \%$ of patients $(46.6 \%, 73.3 \%$ and $100 \%$ of patients with slight, moderate and severe chronic periodontitis, respectively). The genotype fimA was detected in $66 \%$ of positive subjects for $P$. gingivalis, whereas fimA IV and I were the most prevalent genotypes. In addition, fimA IV was detected in $75 \%$ and fimA I in $62.5 \%$ of severe and moderate cases, respectively. Increased levels of fimA IV were associated with severe chronic periodontitis.

Conclusions: These findings suggest that there is a high prevalence of $P$. gingivalis and its fimA IV and I genotypes in chronic periodontitis patients. Furthermore, fimA IV was associated with severe chronic periodontitis in this Chilean population.

KEYWORDS

Chronic periodontitis; Biofilms; Bacterial fimbriae.

Rev. Clin. Periodoncia Implantol. Rehabil. Oral Vol. 10(3); 141-144, 2017.

\section{INTRODUCCIÓN}

Uno de los principales microorganísmos descritos en la etiopatogenia de la enfermedad periodontal y particularmente de periodontitis crónica es Porphyromonas gingivalis ${ }^{(1)}$. Se ha visto que su frecuencia varía según el grupo étnico ${ }^{(2)}$, ubicación geográfica ${ }^{(3)}$ y metodología del estudio(4,5).
$P$. gingivalis es un periodontopatógeno clave en el desarrollo y progresión de la enfermedad periodontal ${ }^{(1,5)}$ debido a que sus factores de virulencia favorecen la adhesión, invasión y destrucción de los tejidos periodontales, pudiendo conducir a la formación de sacos periodontales ${ }^{(7)}$. Las fímbrias de $P$. gingivalis se localizan en la superficie celular y son un 
factor crítico de colonización(8). El gen que codifica las fimbrias es el fimA se clasifica en seis genotipos (I, Ib, II, III, IV, V) en base a la secuencia de sus nucleótidos ${ }^{(9,10)}$. La prevalencia de los diferentes genotipos del fimA ha sido estudiada y muestra una gran variación según la población analizada ${ }^{(11-14)}$. Una serie de estudios han sugerido una mayor prevalencia de fimA II, seguida de fimA IV, en pacientes con periodontitis ${ }^{(11,12,15)}$. Estos dos genotipos han estado estrechamente asociados con la enfermedad periodontal severa ${ }^{(10)}$. Por otro lado, los genotipos fimA I y $\mathrm{V}$ se han asociado con un estado de salud periodontal(15)

La prevalencia de $P$. gingivalis en pacientes chilenos con periodontitis crónica es elevada $(4,16,17)$. Sin embargo, información disponible sobre los niveles de este microorganismo o su distribución según diferentes genotipos fimA en esta población es limitada. El objetivo de este estudio fue cuantificar los niveles de $P$. gingivalis y medir la prevalencia de los seis genotipos fimA en pacientes chilenos con diferentes grado de severidad de periodontitis crónica.

\section{MATERIALES Y MÉTODOS}

Sujetos. Se presenta un estudio de corte transversal. Se invitó a participar en este estudio a cuarenta y cinco sujetos (15 hombres y 30 mujeres, rango de edad de 20-75 años) con diagnóstico de periodontitis crónica y seleccionados por conveniencia según grado de severidad. Los criterios de exclusión incluyeron embarazo, tratamiento periodontal previo y antecedentes de uso sistémico o local de antimicrobianos en los seis meses previos al muestreo, además de condiciones sistémicas conocidas que pudieran influir en las condiciones periodontales. Los pacientes incluidos en el estudio tenían que presentar al menos 5 dientes presentes en boca y ser diagnosticados con periodontitis crónica según los criterios diagnósticos de American Academy of Periodontology en 1999(18).

Evaluación clínica. Se registraron variables socio-demográficas, las que incluyeron edad, sexo, además de la historia médica. Se realizó un examen clínico del estado periodontal. El examen periodontal incluyo la evaluación de la profundidad de sondaje, sangramiento al sondaje y pérdida del nivel de inserción periodontal en seis sitios por diente: mesiobucal, bucal, distovestibular, distolingual, lingual y mesiolingual. Luego, los pacientes se dividieron en tres diferentes categorías de 15 sujetos cada uno según la pérdida de inserción clínica (NIC): leve (1-2 $\mathrm{mm} \mathrm{NIC}$ ), moderada (3-4 mm NIC) y severa (= o> $5 \mathrm{~mm} \mathrm{NIC})^{(19)}$.

Muestras microbiológicas y extracción de ADN genómico. Después del diagnóstico periodontal, se seleccionaron para el muestreo los tres sacos periodontales más profundos de cada paciente, eliminando el biofilm supragingival con una cureta estéril. En el caso de igualdad de profundidad de los sitios se priorizaron según el orden siguiente: molares $>$ premolares $>$ caninos $>$ incisivos. Para la recolección de las muestras subgingivales se insertaron los conos de papel (cono número 35, Dentsplay Maillefer) en los tres sacos periodontales seleccionados durante 10 seg como se ha descrito previamente ${ }^{(14)}$. Luego se transfirieron los tres conos con las muestras de cada paciente inmediatamente a un tubo conteniendo el medio de transporte anaeróbico (ATM AS-911, Anaerobe Systems, Morgan Hill, CA, EE.UU.), el cual fue identificado enviado al Laboratorio de Microbiología Oral de la Universidad de Talca. Las muestras se mantuvieron a $37^{\circ} \mathrm{C}$ durante $15 \mathrm{~min}$, se homogeneizaron y se transfirieron alícuotas de $100 \mu \mathrm{L}$ a tubos que contenían $300 \mu \mathrm{L}$ de agua purificada estéril. Después se lisaron las células y se extrajo el ADN sumergiendo los tubos en agua hirviendo durante $10 \mathrm{~min}$. Finalmente, el sobrenadante se recuperó y se purificó usando el kit de aislamiento de ADN Ultraclean ${ }^{\circledR} 15$ (MO BIO laboratories Inc, Carlsbad, CA, EE.UU.).

Cuantificación de $\boldsymbol{P}$. gingivalis mediante PCR cuantitativa (qPCR) Para cuantificar la carga bacteriana específica de $P$. gingivalis, se amplificó mediante qPCR del gen 16S rRNA. Como templado, se utilizó el ADN genómico bacteriano obtenido desde las muestras clínicas analizadas en éste estudio. Para poder calcular el número de $P$. gingivalis en cada muestra analizada, se obtuvieron curvas de calibración amplificando el gen 16S rRNA desde plásmidos que portan como inserto este gen. Para construir este plásmido, el amplicón para el gen del 16s rRNA se obtuvo a partir del ADN genómico de la cepa de referencia $P$. gingivalis ATCC 33277. Este amplicón se insertó en plásmidos y se clonó en $E$. coli como se ha descrito previamente ${ }^{(20)}$. El ADN del plásmido se extrajo mediante el kit GeneJET ${ }^{\circledR}$ Plasmid Miniprep (Thermo Fisher Scientific) y se usó como molde para obtener las curvas de calibración utilizando el sistema qPCR Mx3000P (Agilent Technologies, Santa Clara, CA, EE.UU). El rango de cuantificación de esta curva de calibración fue de $10^{2}$ a $10^{-}$ copias para este gen, con una sensibilidad de $2,2 \times 10^{2}$ copias en una dilución de $19^{-9}$. La cuantificación de $P$. gingivalis se realizó por qPCR utilizando un set de partidores específicos para amplificar 16S rRNA. Las reacciones de qPCR se realizaron a $20 \mu \mathrm{l}$ de volumen final con $10 \mu \mathrm{L}$ de Brilliant II SYBR ${ }^{\circledR}$ Green QPCR Master Mix (Agilent Technologies), $2 \mu \mathrm{L}$ de ADN molde y $10 \mu \mathrm{M}$ de cada primer. Los parámetros de ciclos térmicos incluyeron desnaturalización inicial a $95^{\circ} \mathrm{C}$ durante $5 \mathrm{~min}$, seguido de 40 ciclos de $95^{\circ} \mathrm{C}$ durante $15 \mathrm{seg}$ y $60^{\circ} \mathrm{C}$ durante $45 \mathrm{seg}$. El tamaño de los productos de qPCR se confirmó por visualización del gel de electroforesis de ADN (gel de agarosa al 1,5\% / bromuro de etidio) y visualización con luz UV. Además, la especificidad del amplicón se corroboró por la curva de disociación obtenida por cada qPCR realizado.

Genotipificación fimA mediante qPCR. Como templado para estas reacciones, se utilizó el ADN genómico bacteriano obtenido desde las muestras clínicas y partidores específicos para amplificar las genovariantes del fimA I, II, III, IV (21), $\mathrm{V}^{(22)}$ y Ib ${ }^{(23)}$. Se siguieron los mismos parámetros de ciclo térmico descrito para la cuantificación de $P$. gingivalis. El tamaño de los amplicones de qPCR obtenidos se veríficó por electroforesis en gel de agarosa, de acuerdo a lo descrito anteriormente. La especificidad de los productos obtenidos se comprobó mediante la curva de disociación que se generó para cada reacción realizada.

Cuantificación de $P$. gingivalis y genotipificación fimA mediante PCR cuantitativa (qPCR). Los plásmidos que contenían los genes diana se amplificaron para crear curvas estándar para la cuantificación. El amplicón de PCR para el rRNA $16 \mathrm{~S}$ se obtuvo a partir del ADN genómico de la cepa de $P$. gingivalis ATCC 33277 . Estos se insertaron en plásmidos y se clonaron en $E$. coli como se ha descrito previamente ${ }^{(20)}$. El ADN del plásmido se extrajo mediante el kit GeneJET ${ }^{\circledR}$ Plasmid Miniprep (Thermo Fisher Scientific) y se usó como molde para obtener las curvas estándar utilizando el sistema qPCR Mx3000P (Agilent Technologies, Santa Clara, CA, EE.UU).

La cuantificación de $P$. gingivalis y genotipificación de fimA fueron realizados por qPCR utilizando set de primers específicos para amplificar 16S rRNA y fimA I, II, III, IV (21) $\mathrm{V}^{(22)}$ y $\mathrm{Ib}^{(23)}$. Las reacciones de $\mathrm{qPCR}$ se realizaron a $20 \mu \mathrm{l}$ de volumen final con $10 \mu \mathrm{L}$ de Brilliant II SYBR ${ }^{\circledR}$ Green QPCR Master Mix (Agilent Technologies), $2 \mu \mathrm{L}$ de ADN molde y $10 \mu \mathrm{M}$ de cada primer. Los parámetros de ciclo incluyeron desnaturalización inicial a $95^{\circ} \mathrm{C}$ durante $5 \mathrm{~min}$, seguido de 40 ciclos de $95^{\circ} \mathrm{C}$ durante 15 seg y $60^{\circ} \mathrm{C}$ durante $45 \mathrm{seg}$. El tamaño de los productos de PCR se confirmó por visualización del gel de electroforesis de ADN (gel de agarosa al 1,5\% / bromuro de etidio) y visualización con luz UV. La cuantificación de $P$. gingivalis y gen fimA se realizó como se ha descrito anteriormente(20).

Aspectos éticos. Este estudio fue llevado a cabo tras la aprobación del protocolo y del consentimiento informado por parte del Comité de Bioética de la Universidad de Talca. Los individuos que participaron de la investigación firmaron, previo a los procedimientos de entrevista y examen clínico, un consentimiento informado y posterior al examen se les informo de su estado de salud periodontal.

Análisis de los datos. La frecuencia y cuantificación de $P$. gingivalis y la frecuencia de cada genotipo $\operatorname{fim} A$ con respecto a la severidad de periodontitis crónica se analizaron con prueba de Chi cuadrado. La significación estadística fue dado por $p$ valor $\leq 0,05$. Los datos se analizaron utilizando el software SPSS 15.0 (IBM, Chicago, IL, EE.UU.)

\section{RESULTADOS}

El estudio fue realizado con 45 sujetos que presentaron periodontitis crónica, sus datos demográficos y parámetros clínicos son presentados en la Tabla 1. Se detectó $P$. gingivalis en 33 sujetos con periodontitis crónica $(73,3 \%)$. Su distribución entre los grupos con diferentes grados de severidad de periodontitis crónica fue estadísticamente significativa $(p<0,05)($ Tabla 2$)$, observándose que $P$. gingivalis estuvo presente en $7(46,6 \%), 11(73,3 \%)$ y $15(100 \%)$ sujetos con las formas leve, moderada y severa de la enfermedad, respectivamente. La cuantificación de este periodontopatógeno indica que el $63,6 \%$ de los sujetos positivos tenían una carga bacteriana $\geq 10^{(5)}$ células $/ \mathrm{mL}$ (Tabla 2). Además, el $80 \%$ de los pacientes con periodontitis crónica severa presentaron una carga bacteriana $\geq 10^{5}$ células $/ \mathrm{mL}$ y estaban relacionados con una mayor pérdida de nivel de inserción clínica y profundidad de sondaje. Por otro lado el $85,7 \%$ los pacientes con periodontitis crónica leve se relacionaron con recuentos bacterianos con valores $\leq 10^{(5)}$ células $/ \mathrm{mL}$.

El gen fimA se detectó en $22(66 \%)$ de los 33 sujetos positivos para $P$. gingivalis. En 14 de estas muestras positivas para este genotipo se detectó sólo un genotipo, mientras que en las otras ocho muestras mostraron una combinación de genotipos diferentes (Tabla 3). Con respecto a la frecuencia de los genotipos fimA, se observó la expresión de fimA IV $(36,4 \%)$ y fimA I $(27,3 \%)$, siendo el primero detectado principalmente en presencia de casos severos de enfermedad periodontal (75\%), lo que indica una asociación estadísticamente significativa $(p<0,05)$ entre este genotipo y la severidad de periodontitis crónica (Fig. 1). Por otro lado el genotipo fimA I fue más frecuentemente detectado en casos donde la periodontitis era moderada $(62,5 \%)$. Los genotipos fimA III y fimA V no fueron detectados en esta población. 
Tabla 1 Características demográficas y parámetros clínicos en relación a severidad de periodontitis crónica.

\begin{tabular}{cccc}
\hline Variables & $\begin{array}{c}\text { Leve } \\
(\mathbf{n}=\mathbf{1 5})\end{array}$ & $\begin{array}{c}\text { Moderada } \\
(\mathbf{n}=\mathbf{1 5})\end{array}$ & $\begin{array}{c}\text { Severa } \\
(\mathbf{n}=\mathbf{1 5})\end{array}$ \\
\hline $\begin{array}{c}\text { sexo } \\
\text { (masculino/feminino) }\end{array}$ & $3 \mathrm{M} / 4 \mathrm{~F}$ & $7 \mathrm{M} / 4 \mathrm{~F}$ & $7 \mathrm{M} / 8 \mathrm{~F}$ \\
\hline $\begin{array}{c}\text { edad } \\
\text { (años) }\end{array}$ & $29,6 \pm 3,3$ & $41,5 \pm 4,0$ & $54,5 \pm 7,2$ \\
\hline $\begin{array}{c}\text { Nivel de inserción } \\
\text { clínica }\end{array}$ & $2,6 \pm 0,5$ & $4,0 \pm 0,7$ & $6,8 \pm 1,8$ \\
\hline $\begin{array}{c}\text { profundidad de } \\
\text { sondaje }\end{array}$ & $3,5 \pm 0,7$ & $4,2 \pm 0,7$ & $5,5 \pm 1,8$ \\
\hline
\end{tabular}

Edad (promedio $\pm D E)$; nivel inserción clínica y profundidad de sondaje ( $\mathrm{mm}$, promedio $\pm D E)$.

Tabla 2 Detección y cuantificación de $P$. gingivalis en relación a severidad de periodontitis crónica.

\begin{tabular}{|c|c|c|c|c|c|}
\hline & & $\begin{array}{l}\text { Leve } \\
\mathrm{n}(\%)\end{array}$ & $\begin{array}{c}\text { Moderada } \\
\text { n (\%) }\end{array}$ & $\begin{array}{c}\text { Severa } \\
\mathrm{n}(\%)\end{array}$ & $\begin{array}{l}\text { Total } \\
\text { n (\%) }\end{array}$ \\
\hline \multirow{3}{*}{ Detección } & Negativo & $8(53,4)$ & $4(26,7)$ & $0(0)$ & $12(26,7)$ \\
\hline & Positivo* & $7(46,6)$ & $11(73,3)$ & $15(100)$ & $33(73,3)$ \\
\hline & Total n (\%) & $15(100)$ & $15(100)$ & $15(100)$ & $45(100)$ \\
\hline \multirow{3}{*}{ Cuantificatión } & $<10^{(5)}$ cells $/ \mathrm{mL}$ & $6(85,7)$ & $3(27,3)$ & $3(20)$ & $12(36,4)$ \\
\hline & $\geq 10^{(5)}$ cells $/ \mathrm{mL}^{*}$ & $1(14,3)$ & $8(72,7)$ & $12(80)$ & $21(63,6)$ \\
\hline & Total n (\%) & $7(100)$ & $11(100)$ & $15(100)$ & $33(100)$ \\
\hline
\end{tabular}

${ }^{*} p \leq 0,05$ entre los diferentes grados de severidad de la enfermedad determinado por test Chi cuadrado.

Tabla 3 Distribución de genotipos fimA en relación a severidad de periodontitis crónica.

\begin{tabular}{|c|c|c|c|c|c|}
\hline & $\begin{array}{c}\text { fimA } \\
\text { genotype }\end{array}$ & $\begin{array}{l}\text { Leve } \\
\mathrm{n}(\%)\end{array}$ & $\begin{array}{c}\text { Moderada } \\
\text { n (\%) }\end{array}$ & $\begin{array}{c}\text { Severa } \\
\text { n (\%) }\end{array}$ & $\begin{array}{l}\text { Total } \\
\text { n (\%) }\end{array}$ \\
\hline \multirow{7}{*}{$\begin{array}{l}\text { Genotipo } \\
\text { Simple }\end{array}$} & 1 & $2(100)$ & $3(37.5)$ & $1(8.3)$ & $6(27.3)$ \\
\hline & Ib & 0 & 0 & 0 & 0 \\
\hline & II & 0 & 0 & 0 & 0 \\
\hline & III & 0 & 0 & 0 & 0 \\
\hline & IV & 0 & $2(25)$ & $6(50)$ & $8(36.4)$ \\
\hline & V & 0 & 0 & 0 & 0 \\
\hline & Subtotal & $2(100)$ & $5(62.5)$ & 7 (58.3) & $14(63.7)$ \\
\hline \multirow{6}{*}{$\begin{array}{c}\text { Genotipo } \\
\text { Combinado }\end{array}$} & I-II & 0 & $2(25)$ & $2(16.7)$ & $4(18.2)$ \\
\hline & Ib-IV & 0 & 0 & $1(8.3)$ & $1(4.5)$ \\
\hline & II-IV & 0 & 0 & 1 (8.3) & $1(4.5)$ \\
\hline & Ib-II-IV & 0 & $1(12.5)$ & 0 & $1(4.5)$ \\
\hline & I-Ib-II-IV & 0 & 0 & $1(8.3)$ & $1(4.5)$ \\
\hline & Subtotal & 0 & $3(37.5)$ & 5 (41.7) & $8(36.3)$ \\
\hline Total n (\%) & & $2(100)$ & $8(100)$ & $12(100)$ & $22(100)$ \\
\hline
\end{tabular}

Cuando una muestra presentó más de un genotipo fimA se detectó principalmente en formas moderadas y severas de periodontitis crónica Observándose que la combinación más frecuente fue fimA I y II $(18,2 \%)$ (Tabla 3). Es importante señalar que fimA IV se detectó con frecuencia en la periodontitis crónica severa $(75 \%)$, lo que indica una asociación estadísticamente significativa $(p<0,05)$ entre este genotipo y la severidad de periodontitis crónica (Fig. 1).

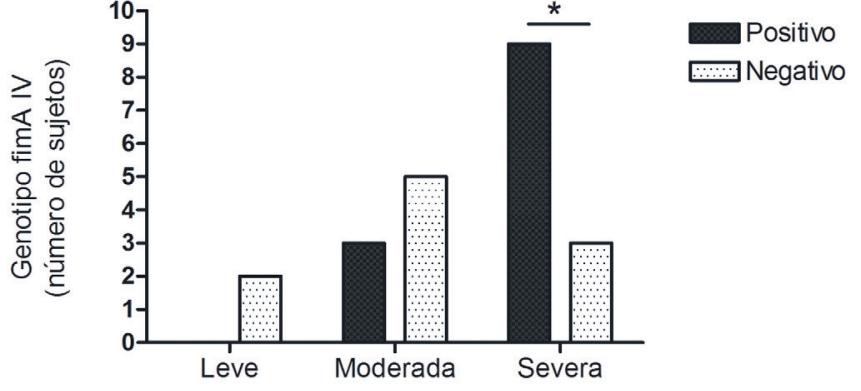

Severidad de la Periodontitis Crónica

Figura 1. Distribución de genotipo fimA IV en relación a severidad de periodontitis crónica. * $\mathrm{p} \leq 0.05$ positivo comparado con negativo determinado por test Chi 2

\section{DISCUSIÓN}

Existe una asociación positiva entre raza / etnia y la composición de la microbiota subgingival en pacientes con enfermedad periodontal ${ }^{(2)}$. Asimismo, los resultados de la literatura sugieren que existiría una variabilidad en la frecuencia de $P$. gingivalis según la localización geográfica observada(3). En Chile, estudios relatan mayor prevalencia de este periodontopatógeno en relación a otros países ${ }^{(5,16)}$. Sin embargo, estos estudios presentan una amplia variación en el rango de sus resultados, reportándose una prevalencia que va desde $11,9 \%{ }^{(5)}$ a $83,8 \%{ }^{(4)}$, en razón de los diferentes protocolos y metodologías empleadas para detectar este patógeno periodontal. El presente estudio buscó no solo detectar sino que también cuantificar los niveles de $P$. gingivalis y la distribución de diferentes genotipos fimA en pacientes con diferentes grados de severidad de periodontitis crónica a través de qPCR. Este método es un procedimiento preciso, sensible y específico, además de permitir la cuantificación confiable de periodontopatógenos en muestras de bioflim subgingival(24).

Nuestros resultados muestran una prevalencia de $73,3 \%$ de este patógeno en pacientes con periodontitis crónica, similar a lo reportado en estudios realizados en $\mathrm{Brasi}^{(25)}$ pero superior a lo reportado en $\mathrm{Holanda}^{(24)}$. Sin embargo, los niveles de P.gingivalis en el biofilm subgingival pueden ser más relevante que su sola detección, puesto que, según nuestros resultados, la cantidad del periodontopatógeno puede variar según la severidad de la enfermedad. De este modo $P$. gingivalis estuvo presente en el $100 \%$ de los pacientes con periodontitis severa, lo cual también fue observado en la población Coreana(26). En este mismo sentido, nuestros datos de cuantificación bacteriana mostraron que la detección de altos niveles de $P$. gingivalis $\left(\geq 10^{(5)}\right.$ células $/ \mathrm{ml}$ ) se asoció con un diagnóstico de enfermedad periodontal severa. Del mismo modo los pacientes con periodontitis leve mostraron niveles más bajos de $P$. gingivalis $\left(\leq 10^{(5)}\right.$ células $/ \mathrm{mL}$ ) lo cual parece reafirmar el rol etiológico de esta bacteria en la destrucción periodontal|(6).

Dentro de los factores que se relaciona directamente con el potencial de virulencia de este periodontopatógeno encontramos el gen fim $A^{(27,28)}$, el cual es un factor de virulencia que otorga diversidad fenotípica y genotípica a las diferentes cepas de $P$. gingivalis. La prevalencia de estos genotipos cambiaría considerablemente entre las diferentes poblaciones ${ }^{(13,14)}$. Nuestro estudio busca relacionar la frecuencia de los distintos genotipos conocidos para fimA en función de la severidad de la periodontitis crónica. Observamos así una alta expresión de los genotipos fimA IV y fimA I (Tabla 3). Estos resultados son consistentes con los estudios que han reportado los genotipos I, II y IV como aquellos más frecuentemente detectados ${ }^{(11,13)}$. Adicionalmente nuestros resultados muestran una asociación entre la detección del genotipo fimA IV y la presencia de periodontitis crónica severa, coincidiendo con los resultados reportados en otros países ${ }^{(20,29,30)}$. Sin embargo, nuestro estudio contrasta con los datos de otras poblaciones en los que el genotipo más frecuente sería el fimA II $(11,13,14,20,21,31,32)$. Estas variaciones pueden ser atribuidas a patrones étnicos y / o geográficos particulares ${ }^{(11,14)}$. Finalmente, una combinación de diferentes genotipos fimA fue detectada en el $36,3 \%$ de los pacientes (Tabla 3); destacando que en siete de los ocho pacientes con genotipos combinados se observó la presencia del fimA II. Esto se relacionó frecuentemente con periodontitis crónica moderada o severa.

Un dato interesante es el hecho de que no se detectaron genotipos fimA en el $34 \%$ de las muestras positivas para $P$. gingivalis. Este número es mayor que los reportados anteriormente ${ }^{(14,15)}$ y podría sugerir la presencia de un genotipo fimA no identificado. Las muestras no identificadas 
se relacionaron principalmente con periodontitis crónica leve, lo que confirmaría la importancia de este gen como factor de virulencia en la patogénesis y progresión de la enfermedad periodontal en la población chilena. Asimismo, otro rasgo que podría caracterizar nuestra población podría ser la ausencia de fenotipos fimA III y V.

Este trabajo muestra que los sujetos chilenos presentan una alta prevalencia de $P$. gingivalis, siendo el genotipo fimA IV el más frecuentemente detectado. A fin de confirmar estos resultados preliminares se sugiere la realización de estudios longitudinales con un mayor número de pacientes. Por otro lado, se recomiendan estudios adicionales en los que se pueda evaluar los diferentes genotipos y su interacción con otros factores de virulencia de este periodontopatógeno.

\section{CONCLUSIONES}

La población estudiada presenta altos niveles de $P$. gingivalis y una alta frecuencia de detección para el genotipo fimA IV, presentándose con mayor frecuencia en pacientes con periodontitis crónica severa. Se evidenció la variabilidad genética de este microorganismo según la severidad de la enfermedad periodontal, siendo aspectos a considerar en la etiopatogenia y en la terapia de la enfermedad periodontal.

\section{CONFLICTO DE INTERÉS}

Todos los autores declaran no presentar conflictos de intereses.

\section{AGRADECIMIENTOS}

Los autores desean agradecer a la Prof. Nora Silva, docente del área de microbiología oral del Dpto. de Patología y Medicina Oral por proporcionar cepas de $P$. gingivalis y Olga Lobos por la ayuda con los procedimientos en el laboratorio de Microbiología, Universidad De Talca.

\section{Bibliografía}

1. Slots J, Ting M. Actinobacillus actinomycetemcomitans and Porphromonas gingivalis in human periodontal disease: occurrence and treatment. Periodonto 2000. 1999;20:82-121

2. Rylev M, Kilian M. Prevalence and distribution of principal periodontal pathogens worldwide. J Clin Periodontol. 2008;35:346-61.

3. Sanz M, van Winkelhoff AJ, Herrera D, Dellemijn-Kippuw N, Simón R, Winke $\mathrm{E}$. Differences in the composition of the subgingival microbiota of two periodontitis populations of different geographical origin. A comparison between Spain and The Netherlands. Eur J Oral Sci. 2000;108:383-92.

4. Herrera D, Contreras A, Gamonal J, Oteo A, Jaramillo A, Silva N, et al. Subgingival microbial profiles in chronic periodontitis patients from Chile, Colombia and Spain. $J$ Clin Periodontol. 2008:35:106-13.

5. Haffajee AD, Bogren A, Hasturk H, Feres M, Lopez NJ, Socransky SS. Subgingival microbiota of chronic periodontitis subjects from different geographic locations. J Clin Periodontol. 2004;31:996-1002.

6. Socransky SS, Haffajee AD. Dental biofilms: difficult therapeutic targets. Periodontol 2000. 2002;28:12-55

7. Fine DH, Kaplan JB, Kachlany SC, Schreiner HC. How we got attached to Actinobacillus actinomycetemcomitans: a model for infectious diseases. Periodontol 2000. 2006;42:114-57.

8. Lamont RJ, Jenkinson HF. Life below the gum line: pathogenic mechanisms of Porphyromonas gingivalis. Microbiol Mol Biol Rev. 1998;62:1244-63.

9. Nakagawa I, Amano A, Ohara-Nemoto Y, Endoh N, Morisaki I, Kimura S, et al Identification of a new variant of fimA gene of Porphyromonas gingivalis and its distribution in adults and disabled populations with periodontitis. J Periodontal Res. 2002:37:425-32.

10. Amano A, Nakagawa I, Okahashi N, Hamada N. Variations of Porphyromonas gingivalis fimbriae in relation to microbial pathogenesis. J Periodontal Res. 2004;39:136-42.

11. Zhao L, Wu YF, Meng S, Yang H, OuYang YL, Zhou XD. Prevalence of fimA genotypes of Porphyromonas gingivalis and periodontal health status in Chinese adults. J Periodont Res. 2007:42:511-17.

12. Puig-Silla M, Dasí-Fernández F, Montiel-Company JM, Almerich-Silla JM Prevalence of fimA genotypes of Porphyromonas gingivalis and other periodontal bacteria in a Spanish population with chronic periodontitis. Med Oral Patol Oral Ci Bucal. 2012;17:e1047-53.

13. Beikler T, Peters U, Prajaneh S, Prior K, Ehmke B, Flemmig TF. Prevalence of Porphyromonas gingivalis fimA genotypes in Caucasians. Eur $\mathrm{J}$ Oral Sci. 2003;111:390-4.

14. Missailidis CG, Umeda JE, Ota-Tsuzuki C, Anzai D, Mayer MP. Distribution of fimA genotypes of Porphyromonas gingivalis in subjects with various periodontal conditions. Oral Microbiol Immunol. 2004:19:224-9.

15. Amano A, Kuboniwa M, Nakagawa I, Akiyama S, Morisaki I, Hamada S. Prevalence of specific genotypes of Porphyromonas gingivalis fimA and periodontal health status. J Dent Res. 2000;79:1664-8.

16. López NJ, Socransky SS, da Silva I, Japlit MR Haffajee AD. Subgingival microbiota of Chilean patients with chronic periodontitis. J Periodontol. 2004;75:717-25.
17. Gajardo M, Silva N, Gómez L, León R, Parra B, Contreras A, et al. Prevalence of periodontopathic bacteria in aggresive periodontitis patients in a Chilean population. J Periodontol. 2005;76:289-94.

18. Armitage GC. Development of a classification system for periodontal diseases and conditions. Ann Periodontol. 1999;4:1-6.

19. Lindhe J, Ranney R, Lamster I, Charles A, Chung CP Flemmig T, et al. Consensus report: Chronic periodontitis. Ann Periodontol. 1999;4:38-38.

20. Teixeira SR, Mattarazo F, Feres M, Figueiredo LC, de Faveri M, Simionato MR, et al. Quantification of Porphyromonas gingivalis and fimA genotypes in smoker chronic periodontitis. J Clin Periodontol. 2009;36:482-7.

21. Amano A, Nakagawa I, Kataoka K, Morisaki I, Hamada S. Distribution of Porphyromonas gingivalis strains with fimA genotypes in periodontitis patients. J Clin Microbiol. 1999;37:1426-30.

22. Nakagawa I, Amano A, Kimura RK, Nakamura T, Kawabata S, Hamada S Distribution and molecular characterization of Porphyromonas gingivalis carrying a new type of fimA gene. J Clin Microbiol. 2000;38:1909-14.

23. Nakagawa I, Amano A., Ohara-Nemoto Y, Endoh N, Morisaki I, Kimura S, et al. Identification of a new variant of fimA gene of Porphyromonas gingivalis and its distribution in adults and disabled populations with periodontitis. J Periodont Res. 2002;37:425-32.

24. Boutaga $K$, van Winkelhoff AJ, Vandenbroucke-Grauls CM, Savelkoul PH. Comparison of real-time PCR and culture for detection of Porphyromonas gingivalis in subgingival plaque samples. J Clin Microbiol. 2003;41:4950-4.

25. Cortelli JR, Cortelli SC, Jordan S, Haraszthy VI, Zambon JJ. Prevalence of periodontal pathogens in Brazilian with aggressive or chronic periodontitis. J Clin Periodontol. 2005;32:860-6.

26. Choi BK, Park SH, Yoo YJ, Choi SH, Chai JK, Cho KS, et al. Detection of majo putative periodontopathogens in Korean advanced adult periodontitis patients using a nucleic acid-based approach. J Periodontol 2000. 2000;71:1387-94

27. Loos BG, Van Winkelhoff AJ, Dunford RG, Genco RJ, de Graaff J, Dickinson DP, et al. A statistical approach to the ecology of Porphyromonas gingivalis. J Dent Res. 1992;71:353-8.

28. Zhang YJ, Yasui S, Yoshimura F, Ishikawa I. Multiple restriction fragment length polymorphism genotypes of Porphyromonas ginigivalis in single periodontal pockets. Oral Microbiol Immunol. 1995;10:125-8.

29. van der Ploeg JR, Giertsen E, Lüdin B, Mörgeli C, Zinkernagel AS, Gmür R. Quantitative detection of Porphyromonas gingivalis fimA genotypes in dental plaque. FEMS Microbiol Lett. 2004;232:31-7.

30. Griffen AL, Lyons SR, Becker MR, Moeschberger ML, Leys EJ. Porphyromonas gingivalis strain variability and periodontitis. J Clin Microbiol. 1999;37:4028-33.

31. Fabruzi S, León R, Blanc V, Herrera D, Sanz M. Variability of the fimA gene in Porphyromonas gingivalis isolated from periodontitis and non-periodontitis patients. Med Oral Patol Oral Cir Bucal. 2013;18 e100-5.

32. Nadkarni MA, Chhour KL, Chapple CC, Nguyen KA, Hunter N. The profile of Porphyromonas gingivalis kgp biotype and fimA genotype mosaic in subgingival plaque samples. FEMS Microbiol Lett. 2014;361:190-4. 